\title{
Cascading of Fluctuations in Interdependent Energy Infrastructures: Gas-Grid Coupling
}

\author{
Michael Chertkov ${ }^{a, b, *}$, Scott Backhaus ${ }^{c, b}$, and Vladimir Lebedev ${ }^{d}$ \\ ${ }^{a}$ Theoretical Division and Center for Nonlinear Studies, Los Alamos National Laboratory, NM 87545, USA \\ ${ }^{b}$ New Mexico Consortium, Los Alamos, NM 87544, USA \\ ${ }^{c}$ Materials, Physics \& Applications Division, Los Alamos National Laboratory, NM 87545, USA \\ ${ }^{d}$ Landau Institute for Theoretical Physics, 142432, Moscow Region, \\ Chernogolovka, Akademika Semenova av., 1-A, Russia and \\ * Corresponding author [ e-mail: chertkov@lanl.gov] \\ (Dated: July 30, 2015)
}


10 Abstract:

11 The revolution of hydraulic fracturing ${ }^{1}$ has dramatically increased the supply and lowered the cost of natural gas in the United 12 States driving an expansion of natural gas-fired generation capacity in many electrical grids ${ }^{2}$. Unrelated to the natural gas 13 expansion, lower capital costs ${ }^{3}$ and renewable portfolio ${ }^{4}$ standards are driving an expansion of intermittent renewable generation 14 capacity such as wind and photovoltaic generation. These two changes may potentially combine to create new threats to the 15 reliability of these interdependent energy infrastructures. Natural gas-fired generators are often used to balance the fluctuating 16 output of wind generation. However, the time-varying output of these generators results in time-varying natural gas burn rates 17 that impact the pressure in interstate transmission pipelines. Fluctuating pressure impacts the reliability of natural gas deliveries 18 to those same generators and the safety of pipeline operations. We adopt a partial differential equation model of natural gas 19 pipelines and use this model to explore the effect of intermittent wind generation on the fluctuations of pressure in natural gas 20 pipelines. The mean square pressure fluctuations are found to grow linearly in time with points of maximum deviation occurring 21 at the locations of flow reversals.

22 Keywords:

Natural Gas Network, Power Grid Network, Optimization, Uncertainty, Fluctuations

24

25

26 The ongoing evolution to intermittent wind and solar electric generation is causing many electrical grid operators to use more agile natural gas-fired electric generation to balance these new stochastic resources. This interdependence causes a cascade of

${ }^{27}$ the fluctuations of renewable generation into the systems that supply fuel to the gas-fired generators, i.e. natural gas pipelines. We develop a model of the coupling between electrical grid fluctuations and natural gas pipeline systems, analyze the resulting

${ }^{28}$ fluctuations of pipeline pressure, and draw conclusions about the impact of renewable electrical generation on the stability and ${ }_{29}$ security of natural gas pipelines.

By making unconventional natural gas sources economic to extract, hydrofracking has created a revolution in the U.S. natural gas industry ${ }^{1}$. Many of these new gas sources are in nontraditional locations such as the Marcellus shale in Pennsylvania, the ${ }_{30}$ Niobrara shale in Eastern Colorado, and the Bakken shale in North Dakota. See Fig. 1. The dramatic increase in supply has driven down prices and spurred many new or expanded uses for natural gas ${ }^{5,6}$. This revolution in the natural gas supply and loads is creating new challenges for natural gas pipelines that transport the gas from source to load. With a limited amount of ${ }^{31}$ throughput and short-term gas storage (in the form of pressure in pipeline itself), these pipelines may become vulnerable as their 32 operating environment changes.

${ }^{33}$ A dominant new load on the gas pipelines is natural gas-fired generators. Previously, the marginal cost of electricity from ${ }^{34}$ these generators was higher than from coal-fired generators. However, the rapid drop in gas prices has made gas generation ${ }^{35}$ competitive with coal and spurred its construction. An example of this dramatic expansion is in the electrical grid controlled by ${ }^{36}$ the Independent System Operator of New England (ISO-NE) where natural gas-fired electrical generation increased from 5\% of ${ }^{37}$ total capacity to $51 \%$ in a span of 20 years $^{2}$. A parallel development in many U.S. electrical grids is the expansion of intermittent ${ }^{38}$ renewable generation such as wind and PhotoVoltaic (PV) generation-a trend that is expected to continue as utilities work ${ }^{39}$ to meet state-imposed renewable portfolio standards that mandate a certain fraction of electrical generation be derived from ${ }^{40}$ renewable sources. See Fig. 2. In contrast to traditional nuclear, coal, or gas-fired generation, these new forms of generation ${ }^{41}$ have a small degree of controllability. To maintain the second-by-second balance of generation and load, other grid resources ${ }^{42}$ must respond to counteract the fluctuations of the intermittent generation. Although many different types of advanced control 43 of nontraditional resources are being considered to provide these balancing services, e.g. grid-scale battery storage and demand ${ }^{44}$ response, the currently most available resources are the controllable traditional generators with gas-fired generators being the ${ }^{45}$ most flexible among these.

46 The combination of expanded natural gas-fired generation and its increased use to balance intermittent renewable generation ${ }_{48}^{47}$ is creating loads on natural gas pipelines that are significantly different than in the past. Traditional gas pipeline loads (Load 

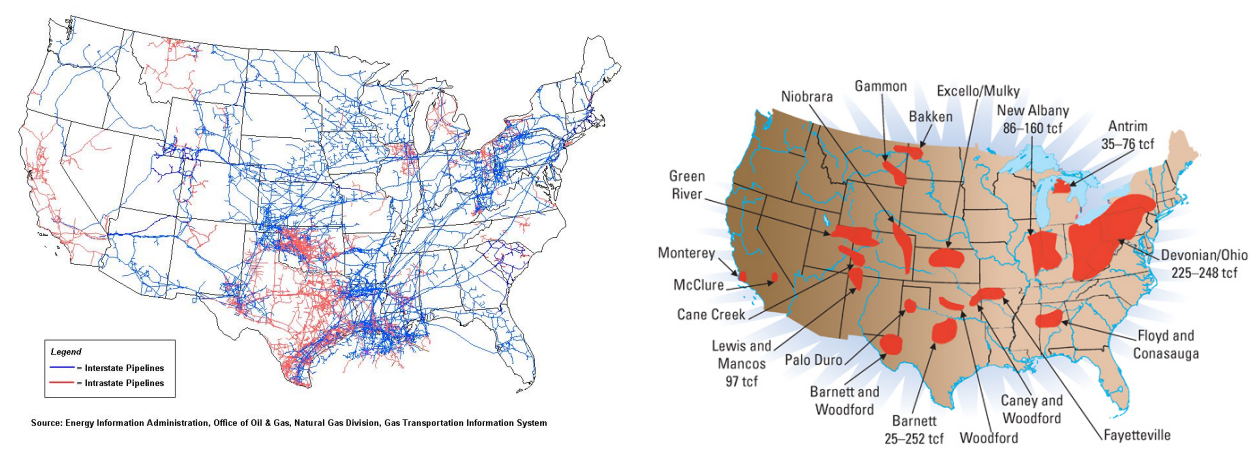

FIG. 1. (Left) The Natural Gas Pipeline Network of the United States. Interstate pipelines are not significantly meshed and primarily display a tree-like structure. (Right) Major US shale gas basins - new sources of natural gas that will encourage realignment of US National Gas Network.
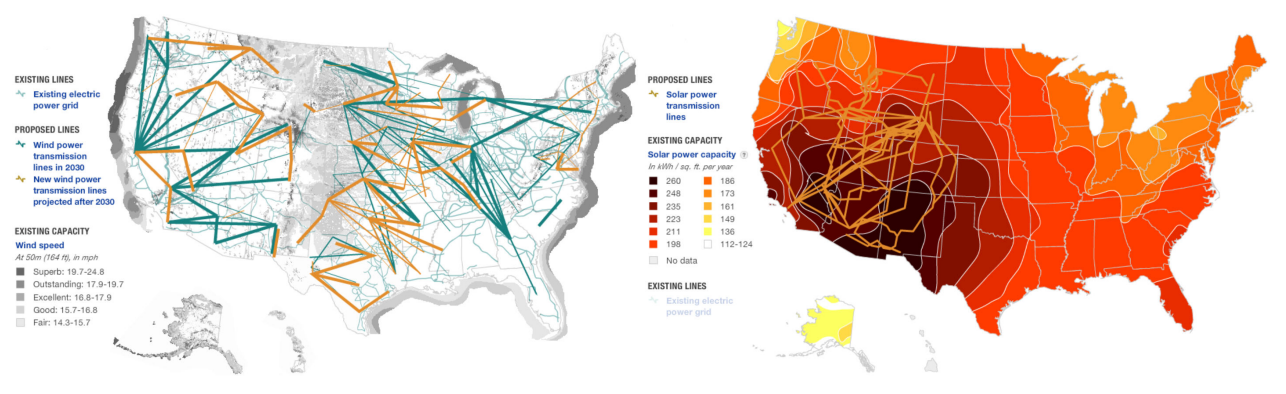

FIG. 2. (Left) US Power Transmission Grid (including potential future transmission expansions) superimposed on wind power capacity map. (Right) Solar power capacity map with proposed transmission lines to improve the integration of solar resources into the existing power grid. (Adapted from National Public Radio, Visualizing the U.S. Electric Grid, 2009.)

59 Distribution Companies or LDCs) primarily serve space or water heating or other individual customer needs and evolve slowly 60 throughout the day in a relatively well-known pattern that can be predicted based on historical information and weather forecasts. ${ }_{61}$ Other traditional pipeline customers are industrial loads that, although they may change from day to day, are very predictable 62 over the span of a day. In contrast, when gas-fired generation is used to balance fluctuating renewable generation, a component of ${ }_{63}$ the resulting gas loads take on a stochastic nature. Unlike the gas load of an LDC, wind and PV generation respond to short-term ${ }_{64}$ fluctuations in environmental conditions, e.g. wind fluctuations on the timescale of 10-100 minutes and solar insolation fluctu65 ations on the timescale of 1-100 minutes. At the longer timescales, these fluctuations may contain spatiotemporal correlations ${ }_{66}$ that increase the aggregate fluctuations of wind or PV generation across an entire electrical grid magnifying the fluctuations of 67 natural gas loads used by gas generators to balance these changes.

68 Fluctuating gas loads create new dynamics in natural gas pipelines that can impact their reliability and the reliability of all ${ }_{69}$ interdependent infrastructures, including the electrical grid. To a great extent, electrical grid dynamics are determined by the very 70 small amount of energy stored in the rotating kinetic energy of large centralized generators. Under a serious upset, this energy 71 storage can maintain the reliable operation of the grid for a second or two while other resources are adjusted to compensate72 typically an adjustment of mechanical power input to these same generators supported by a change in fuel burn rate. In some 73 respects, if the grid "storage" is sufficient to survive the initial upset, an electrical grid with fuel-burning generators has very 74 large amounts of storage on longer timescale in the fuel supplied to those generators.

75 Gas pipelines dynamics evolve on very different timescales. In the short term (10-100 minutes), gas pipelines have a large 76 amount of storage in the compressed natural gas in the pipeline itself. A typical gas pipeline might be run very near its upper 77 limit on pressure of 800 psi whereas the minimum gas delivery pressure is 500 psi. Even if all gas injections into the pipeline 78 were cutoff, the gas loads would slowly reduce the pressure of compressed gas over a few hours without any significant impact 79 on the loads until the gas pressure falls below the minimum delivery pressure. However, unlike the electrical grid, there may 80 be very little gas storage on longer timescales. Injections of gas into the pipeline are scheduled via bi-lateral transactions in gas ${ }_{81}$ markets and are typically held constant throughout a 24-hour period. Therefore, the gas pressure in the pipeline evolves over 82 both space and time according to the spatiotemporal arrangements of the gas injection and gas loads. If the injections and loads вз are out of balance, the gas pressure will undergo spatiotemporal evolution. However, the fluctuations in pressure will not be 
${ }_{84}$ spatially uniform. In fact, the pressure fluctuations are nonlocal with the swings at one locations depending on the behavior at 85 all other locations.

86 The feedback between fluctuating gas loads and gas pressure creates coupled reliability concerns across the natural gas pipeline 87 and electrical grid infrastructures. The nonlocal effects mentioned above can couple with spatiotemporal correlations in the 88 fluctuations of renewable generation through the response of the gas generators to magnify pressure fluctuations at certain 89 locations in the pipeline. These fluctuations may lead to significant over or under pressures, both of which have serious impact 90 on the reliability and safety of the pipeline itself. Under pressures may impact the gas generators by forcing them to reduce 91 electrical output or potentially shutdown to preserve the integrity of service to other pipeline customers. As we will show in this 92 manuscript, the most sensitive locations are those of zero flow at the end of the pipeline with unidirectional flow or at location(s) 93 of flow reversal in pipelines with well separated injection locations. Therefore, the evolving spatial dependence of U.S. natural 94 gas supply will couple to the stochasticity to create additional uncertainty in the reliability of the gas and electrical systems.

${ }_{95}$ Neither gas pipeline nor electrical grid operators have the analysis tools to sufficiently address the probabilistic nature of the 96 reliability impacts created by the coupled stochasticity of these infrastructures. The goal of the manuscript is to lay the foundation 97 for these tools by developing a model and analysis to predict the spatiotemporal evolution of the probability distribution of 98 gas pipeline pressure fluctuations. This first step seeks to develop a measure of probabilistic risk that can be subsequently 99 integrated into the operations of both the electrical and pipeline infrastructure networks. We approach the problem by adopting 100 phenomenological gas flow equations consisting of Partial Differential Equations (PDE) in one spatial dimension that have been 101 accepted as accurate representations of long natural gas pipelines ${ }^{7-11}$.

102 We develop models of fluctuations of gas-fired generator natural gas loads and the constraints imposed by natural gas markets 103 to analyze the stochastically-driven PDEs. We develop analytic expression for probability distributions of gas pipeline pressures 104 as a function of space and time and as a function of the settings of gas compressor stations that push the gas along the pipeline. 105 Our analysis shows that, under constant compressor station settings, the mean square pressure fluctuations grow linearly in time 106 similar to a diffusive process. We find that the largest mean square pressure fluctuations occur at location of zero flow that can 107 potentially occur at any location along the pipeline depending on the average natural gas injections and loads. The results form 108 the basis for a risk-aware optimization problem for the gas compressor stations controls.

109 The material in the rest of the manuscript is organized as follows. Section II reviews the state of the art in modeling gas \& 110 grid coupling and impact of wind generation on the gas network. Section III describes the basic model of natural gas pipelines. ${ }_{111}$ Section IV describes pressure sensitivity to fluctuating gas draws. Future work and extensions are discussed in Section V. 112 Appendixes describe in greater detail the physical models of gas flow and the approximations used to develop the models 113 discussed in the main text.

\section{SYNOPSIS OF THE STATE OF THE ART}

\section{A. Modeling of the gas \& grid coupling}

116 Numerous studies have considered combined optimization and operational planning for interacting energy infrastructures. 117 Integrated natural gas and electric optimal power flow ${ }^{12,13}$ and optimal unit commitment with natural gas security constraints ${ }^{14-16}$ 118 have been proposed, in addition to techniques for short-term operation ${ }^{17-21}$ and expansion planning ${ }^{22,23}$. Those studies rely on 119 the steady-state Weymouth equations ${ }^{24,25}$, which do not capture the dynamic fluctuations that lead to intra-day gas supply issues. 120 Studies on coordinated multi time-period scheduling have also relied on steady-state equations ${ }^{26}$, as well as on finite-difference 121 approximations $^{27,28}$. This is a vibrant field of research where many new ideas continue to emerge. For example, ISO-like natural 122 gas coordinator was suggested $\mathrm{in}^{29}$ to harmonize gas and power industries and enable responsive coordination. Notice that to 123 verify this and other suggestions in a meaningful way further development of optimization and control techniques that utilize 124 physically realistic models of gas network flow transients on the time-scale of power system operations are needed.

\section{B. Wind Variability Impacts on Gas Supply Systems}

126 Several studies have examined the impact of wind variability on the operation of natural gas infrastructure networks ${ }^{20,30}$, 127 which used the so-called "Panhandle A" approximation and the Weymouth equation, respectively, for gas system simulation? . ${ }_{128}$ Similar approaches have been used to examine the impact of stochastic variability on gas systems due to other energy sources 129 with multi-scale behaviors, such as hydro power ${ }^{17}$. A recent study has examined risk assessment for integrated electric power 130 and natural gas systems and proposed techniques for obtaining gas system security certificates within the electric generator 131 dispatch space $^{31}$. 


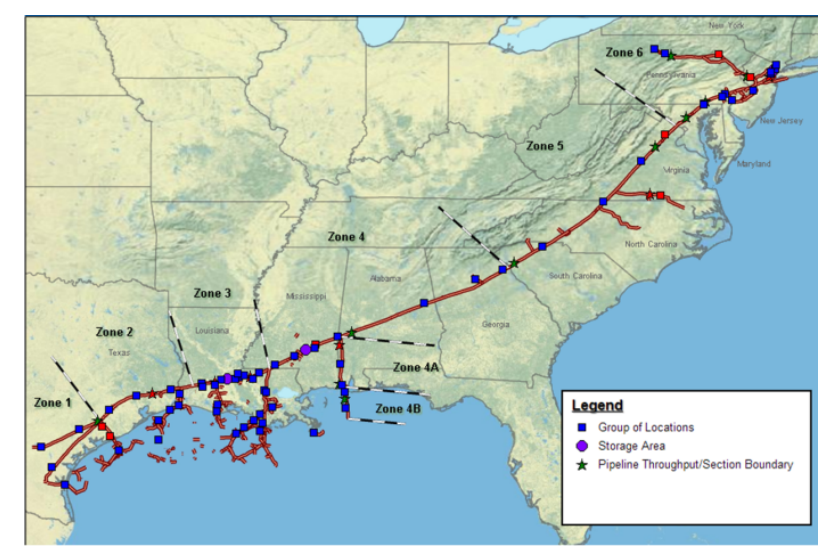

FIG. 3. Schematic representation of the Transco gas transmission network.

${ }_{133}$ The Transco pipeline (see Fig. 3) is a major interstate pipeline that delivers large quantities of natural gas to population centers ${ }_{134}$ and to natural gas-fired generators that supply electricity to those same population centers. Like many other major interstate 135 pipelines, the Transco pipeline displays a nearly radial structure and it is equipped with many compressors that are often nearly 136 equally spaced along its length ( $\sim 50-100 \mathrm{~km}$ between compressors). These two properties are reasonably well approximated by ${ }_{137}$ the radial, distributed compression model discussed in the main text. Under these two approximations, the Transco and similar ${ }_{138}$ pipelines can be analyzed using the simplified models and analysis discussed in the following.

${ }_{139}$ We adopt a phenomenological, spatially one-dimensional model of a transmission pipeline delivering gas over long distances ${ }_{140}(\sim 1000$ 's of $\mathrm{km})$ - a reasonable model of interstate pipelines in the US. The form of this model is generally accepted as 141 an accurate representation of long pipelines ${ }^{7-11}$. See the supplementary information (Appendixes) for model derivation and 142 additional details. The gas injections may be configured in many different ways, e.g. at a single source at the originating end of 143 the pipeline, two sources at either end of the pipeline, or in a distributed manner along the pipeline. However, in all that follows, 144 the injections will be assumed to be constant in time-a simplification that is also a close representation of pipeline operations in 145 the U.S. Natural gas loads are distributed along the pipeline and may fluctuate in time. Pressure gradients drive the gas along 146 the pipeline from sources to sinks, and these gradients are maintained by gas compressors. A few other assumptions in the 147 derivation and analysis of the model are made, but these are mostly taken to simplify the presentation. We will point out where 148 these assumptions can be removed via more complicated analysis.

150 By integrating over the cross section of the natural gas pipeline, the three-dimensional equations of hydrodynamics are reduced 151 to a representation in one spatial dimension. Mass conservation becomes

$$
c_{s}^{-2} \partial_{t} p+\partial_{x} \phi=-q,
$$

152 where $t$ is time, $x$ is coordinate along the pipe $(0<x<L), p$ is the pressure along the pipe, $\phi$ is the mass flow along the pipe, ${ }_{153} q(x)$ is the density of the distributed gas consumption ( $q>0$ for injection and $q<0$ for loads), and $c_{s}$ is sound velocity of the ${ }_{154}$ gas. Using a friction factor $\beta$ as a phenomenological representation of turbulent drag, Navier-Stokes equation becomes

$$
\partial_{x} p+\frac{\beta}{2 d} \frac{\phi|\phi|}{p}=\gamma p
$$

${ }_{155}$ Here, $d$ is the pipe diameter and $\gamma(x)$ is a distributed representation of the many compressor stations in long pipelines. A real ${ }_{156}$ compressor station can operate in several different modes, one of which is a fixed compression ratio $\gamma$ such that $p_{\text {out }}=\gamma p_{\text {in }}$ ${ }_{157}$ where $p_{\text {out }}$ and $p_{\text {in }}$ are the pressures at the outlet and inlet of the compressor. Here, we have distributed this lumped compression ${ }_{158}$ ratio along the pipeline such it makes a positive contribution to the $\partial_{x} p$ of size $\gamma(x)$. Fast acoustic transients are ignored in Eq. (2) 159 by assuming $c_{s} \gg u$, where $u$ is the typical gas velocity. $u$ is generally small enough that this condition holds everywhere in 160 the pipeline, however, $u$ (and its associated Reynolds number) is also large enough that $\beta$ can be taken to be constant. We note 161 that Eqs. $(1,2)$ have already been supplemented with an ideal gas isothermal equation of state for the natural gas of the form ${ }_{162} p=c_{s}^{2} \rho$. (See e.g. ${ }^{32}$ for modern discussion of the modeling and simulations in the general non-isothermal case.) The model 
163 derivation in the Appendixes addresses more general and more realistic settings such as meshed networks and compression 164 spatially concentrated at the nodes.

\section{B. Simplified Market Model}

166 The flow of natural gas in a pipeline is scheduled via bilateral transactions between buyers and sellers in a day-ahead market 167 with market clearing and gas flows scheduling done well in advance of the following 24-hour period of gas delivery. Scheduling 168 consists of determining the locations and constant rates of gas injections. The gas pipeline operator expects that gas loads will 169 be fairly uniform over the 24-hour delivery period. Some level of fluctuating gas load is allowed, and it is these fluctuations that 170 is expected to grow as natural gas-fired electrical generation is increasingly used to balance renewable fluctuations. After the 171 24-hour delivery period begins and gas buyers have better estimates of their actual needs, they can make mid course corrections 172 by transacting and scheduling gas flows in two subsequent intra-day markets at 10 and 14 hours after the start of the 24-hour 173 delivery period. In the three intervening periods, the gas injections are held relatively constant, and it is these periods we seek to 174 analyze.

175 We model these subperiods by first solving for a stationary solution where time-averaged gas loads $q^{(\mathrm{st})}(x)$ are given and are 176 globally balanced by time-independent gas injections at either end of the pipeline, i.e. $\phi^{(\mathrm{st})}(0)=\phi_{0} \geq 0$ and $\phi^{(\mathrm{st})}(L)=\phi_{L} \leq$ 1770 . The stationary gas flow along the pipe is related to the loads by

$$
\partial_{x} \phi^{(\mathbf{s t})}(x)=-q^{(\mathbf{s t})}(x),
$$

178 and the global mass balance implies

$$
\phi^{(\mathrm{st})}(0)-\phi^{(\mathrm{st})}(L)=\int_{0}^{L} d x q_{0}(x) .
$$

179 Natural gas pipeline operators require that Eq. (4) be satisfied over the 24-hour delivery period. To insure this condition over the 180 24-hour period, there may be some deviation in the balance in the intra-day periods to compensate for inaccurate forecasting or 181 changes in the average gas loads. However, in the remainder of this discussion, we will assume that the stationary solution is 182 balanced in each intra-day subperiod. In the following, we will add fluctuations to $q^{(\mathrm{st})}(x)$, and therefore $\phi^{(\mathrm{st})}(x)$, to model the ${ }_{183}$ affects of renewable generation on the pipeline pressure fluctuations.

\section{Compressor Model For Stationary Flows}

185 Before adding fluctuations, we first describe the control of the gas compressors for the stationary gas flows. If the gas loads ${ }_{186} q(x)$ and flow $\phi(x)$ were actually stationary, then the control for the gas compression stations could be computed once and 187 implemented for the entire 24-hour gas delivery period, or at least for the intra-day periods. Natural gas pipeline operators seek 188 to maintain a relatively uniform pressure profile up to the the pressure drop between compressor stations. Our simple model 189 of spatially-distributed compression $\gamma(x)$ in Eq. (2) is a reasonable representation of gas pipeline operations and provides a 190 spatially uniform pressure $p_{0}$ when

$$
\gamma(x)=\frac{\beta \phi^{(\mathrm{st})}(x)\left|\phi^{(\mathrm{st})}(x)\right|}{2 d p_{0}^{2}} .
$$

${ }_{191}$ We pick this model for ease of presentation. Spatially discrete compression and nonuniform pressure profiles ${ }^{33-36}$ can be incor192 porated in an edge-node network model in straightforward manner. See Appendixes for additional discussions.

\section{PRESSURE SENSITIVITY TO FLUCTUATING GAS DRAWS}

194 Time-dependent gas loads require the solution of the dynamic versions of Eqs. $(1,2)$. Here, we consider the time-dependent 195 component to be fluctuations of the gas loads about their forecasted values, $q(t ; x)=q^{(\mathrm{st})}(x)+\xi(t ; x)$, where $\xi(t ; x)$ models the 196 random, zero mean and statistically stationary fluctuations. As described in Appendix B when these fluctuations are relatively 197 weak (even though they may be spatio-temporarily nontrivial), an analytical solution for the time-dependent variance of the gas 198 pressure valid at $t \gg \tau$ becomes

$$
\frac{\left\langle(\delta p(x))^{2}\right\rangle}{p_{0}^{2}}=\left(\frac{\bar{q}^{(\mathrm{st})} c_{s}^{2} \tau}{p_{0}^{2}}\right)^{2} \frac{t}{\tau}\left(\frac{Z(x)}{Y}\right)^{2} \iint_{0}^{L} \frac{d x_{1} d x_{2}}{L^{2}} \frac{\left\langle\xi\left(t, x_{1}\right) \xi\left(t, x_{2}\right)\right\rangle}{\left(\bar{q}^{(\mathbf{s t})}\right)^{2}} .
$$




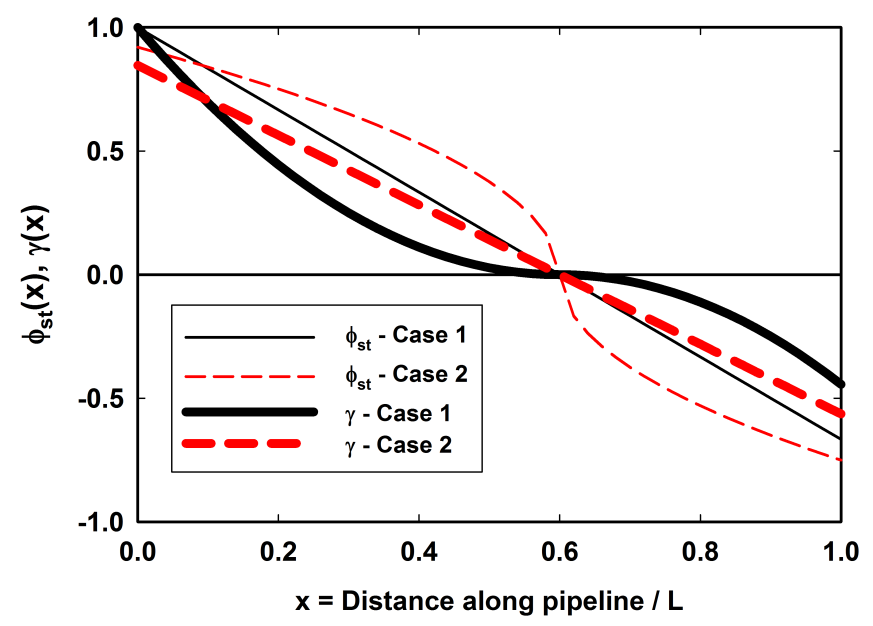

FIG. 4. Stationary mass flux $\phi^{(\mathrm{st})}(x)$ and compression $\gamma(x)$ though the pipeline versus the distance $x$ along the pipeline. Here, the length of the pipeline as been set to one, i.e. $L=1$. The plot shows two different cases of stationary mass flux to demonstrate the effect on the spatial dependence of the sensitivity parameter $Z(x) / Y$. In both cases, the flow reversal occurs at $x^{*}=0.6$. The combined mass flux into the pipe from $x=0$ and $x=1$ is the same for both cases. Case 1 is comprised of uniformly distributed gas loads at all locations along the pipeline. Case 2 represents a combination of some distributed load along with a concentrated load at $x=x^{*}=0.6$.

199 The solution shows that a pipeline's sensitivity to fluctuating gas draws depends on the stationary solutions $\phi^{(\mathrm{st})}(x)$ or $\gamma(x)$ and 200 the statistics of the fluctuating gas loads.

201 Here, we analyze three exemplary stationary configurations to explore the qualitative features of this sensitivity. The first two 202 cases are shown in Fig. 4. ${ }_{215} C \equiv \beta \phi_{0}^{2} L /\left(d p_{0}^{2}\right)$, that scales the compression density $\gamma(x)$. Interpreting the distributed compression in terms of a set of ${ }_{216}$ discrete compressor stations of uniform compression ratio, Case 2 with $C=50$ corresponds to between 6 and 7 compressors ${ }_{217}$ with compression ratio 1.5 placed uniformly between $x=0$ to $x_{*}$, i.e. a typical number of compressors for a mildly stressed 218 pipeline configuration.

${ }_{219}$ When the pipeline is under very little stress $(C=1)$, both Case 1 and Case 2 show a relatively uniform $Z(x) / Y \sim 1$. Under 220 these conditions, there are no regions of the pipeline that show a significantly enhanced sensitivity to stochastic gas loads. As 221 the stress is increased (larger $C$ ), proportionally more compression is deployed in the stationary solution. Both Case 1 and Case 2222 show a depression of $Z(x) / Y$ near the ends of the pipeline, i.e. the injection points, and an enhancement near $x_{*}$. Although ${ }_{223}$ the total gas injection into the pipeline is the same in Cases 1 and 2 (for the same value of $C$ ), the enhancement of the sensitivity 224 to stochastic gas loads in Case 2 is stronger and more focused for two reasons. First, the total (aggregated) compression on the 225 system is larger in Case 2. This can be seen from the curves for compression $\gamma(x)$ in Fig. 4. Second, the stationary gas loads are 226 more concentrated near $x_{*}$ resulting in more compression located near $x_{*}$. After normalization by $Y, Z(x) / Y$ displays a sharper 227 peak. If the gas load were entirely concentrated at $x_{*}$, the mass flux and compression would be uniform along the pipeline (on 228 either side of $x_{*}$ ), and the peak in $Z(x) / Y$ would be even sharper. 


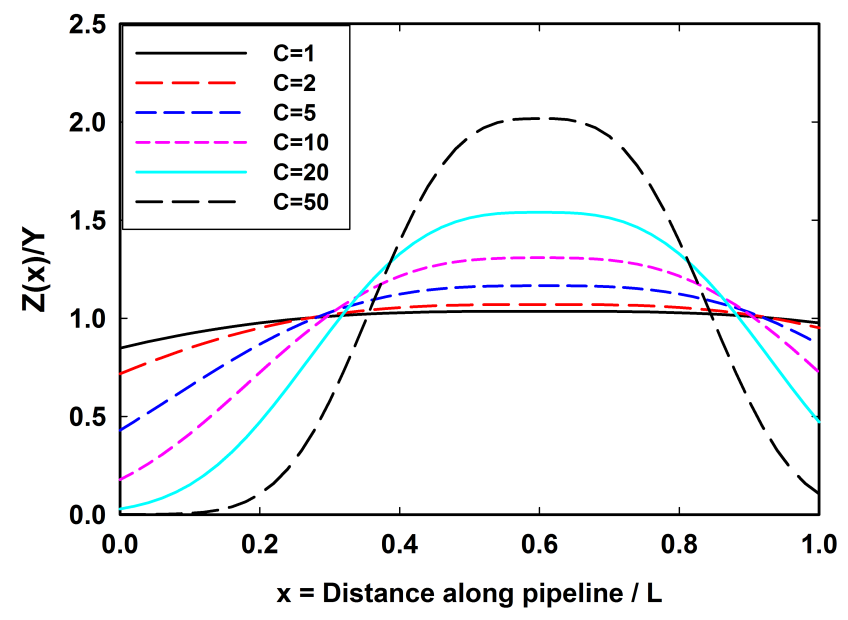

FIG. 5. The fluctuation sensitivity parameter $Z(x) / Y$ versus $x$ for Case 1 in Fig. 4 . For a given pipeline geometry, the different curves represent different scalings of the total stationary mass flux into the pipeline or the total compression deployed in the stationary solution.

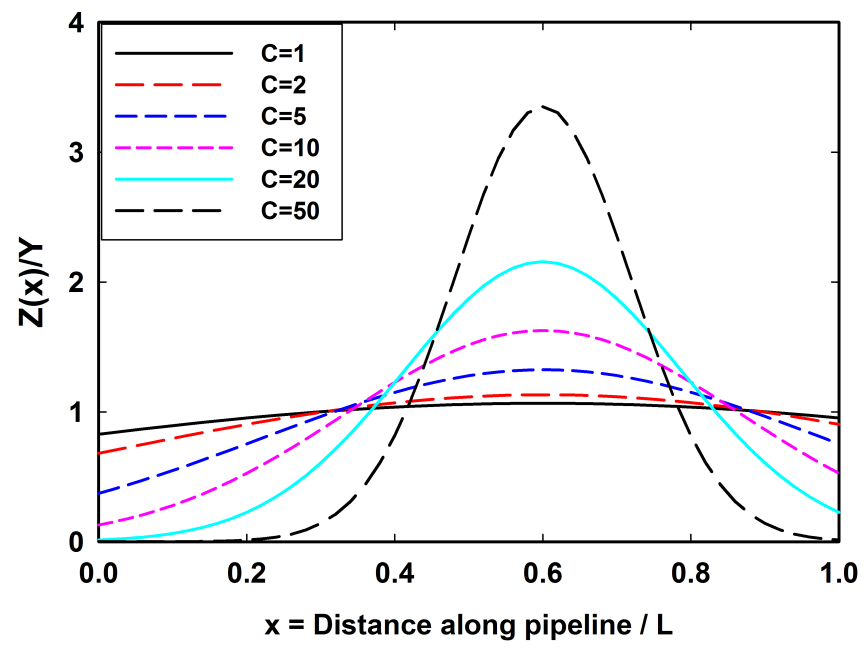

FIG. 6. Same as Fig. 5 except for Case 2 in Fig. 4.

The result that the pipeline shows the highest sensitivity to fluctuations near $x_{*}$ is not a coincidence. The mass flux in the 230 pipeline exhibits a reversal at this point and the compression changes sign. It is at the flow reversal that the integral in Eq. (6) of ${ }_{231}$ the Appendix is the largest. Therefore, in pipelines where the direction of the stationary mass flux is primarily in one direction 232 over long distances, the resulting compression will cause the points of flow reversal to be the most sensitive to pressure fluctuation ${ }_{233}$ from stochastic gas draws. This qualitative result begins to suggest the possibility of fluctuation-aware control algorithms that ${ }_{234}$ adjust either the mean gas pressure or the spatial distribution of compression to limit the probability of the gas pressure violating 235 either upper or lower pressure limits.

236 Gas injections at the ends of the pipeline do not always dominate the flow in a pipeline. Such a situation may occur near the ${ }_{237}$ beginning of a major pipeline where there are many sources of gas injections interspersed with many gas customers. The flow 238 in the pipeline may alternate many times before a significant unidirectional flow builds up. This situation often occurs in the 239 Williams Transco interstate pipeline near its beginning in Texas ${ }^{37}$. This situation may also arise in smaller intrastate pipelines 240 where many smaller, spatially distributed sources are injecting into a pipeline that is serving many different customers. Case 3 241 models these configurations by distributing both gas loads and injections along the pipeline with zero injection or load at the 242 ends, i.e. $\phi^{(\mathrm{st})}(0)=\phi^{(\mathrm{St})}(L)=0$. Fig. 7 (solid line) displays a realization of spatially disordered stationary loads and injections 


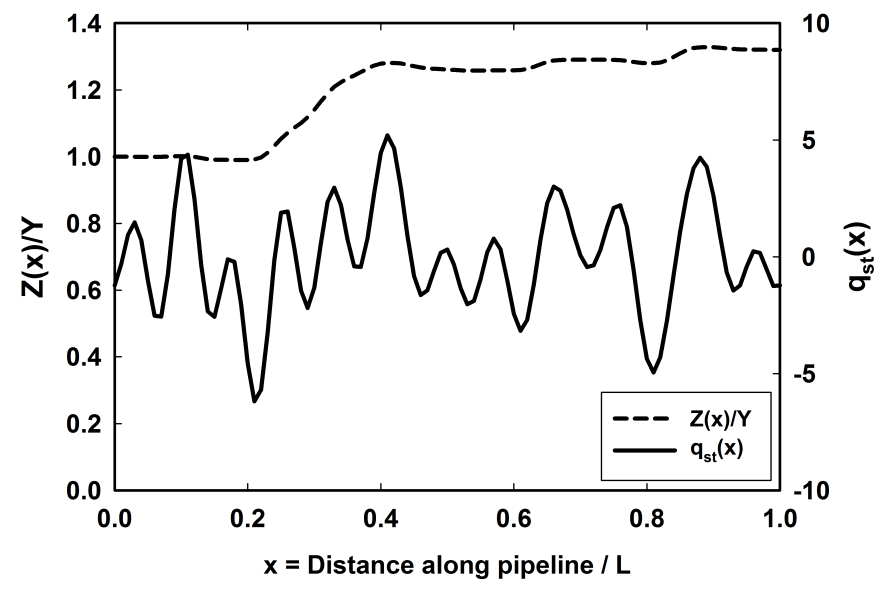

FIG. 7. Disordered stationary gas injections and loads $\left(q^{(\mathrm{st})}\right.$, solid line) and the fluctuation sensitivity parameter $(Z(x) / Y$, dashed line $)$ versus $x$ for Case 3. The total gas injection into the pipeline is equivalent to $C \approx 14.9$ in Case 1 in Fig. 5 or Case 2 in Fig. 6. The disorder of $q^{(\mathrm{st})}(x)$ results in many flow reversals that suppress $Z(x) / Y$ suggesting that this configuration is more robust to gas load fluctuations than a pipeline with more unidirectional flow.

${ }_{243} q^{(\mathrm{st})}(x)$ that corresponds to a total gas flow equivalent to $C \approx 14.9$ in Case 1 or 2 from above. Although the total gas injection 244 is similar, the frequent flow reversals limit and the build up of the integral in Eq. (6) reduces the values of $Z(x) / Y$ in Fig. 7 245 (dashed line) as compared to the Cases 1 and 2 where the flow is more spatially uniform. The spatial disorder of $q^{(\mathrm{st})}(x)$ results 246 in a system that is more robust to fluctuations of gas loads.

\section{PERSPECTIVES}

248 We have developed a dynamical model of natural gas pipelines that incorporates the effect of fluctuating gas injections and 249 loads on the pressure at all points along the pipeline. The model divides the injections and loads into a stationary component and 250 a fluctuating in time component. Compressors along the pipeline are adjusted so that the solution for the stationary gas pressure 251 is spatially uniform. An asymptotic solution for the fluctuating pressure factorizes into a product of two terms. The first term 252 depends on the profile of the stationary injection/consumption along the paper and is related to the compression deployed in the ${ }_{253}$ stationary solution. Surprisingly, this term does not depend on the gas load fluctuations. The second term grows diffusively in ${ }_{254}$ time as given by a spatiotemporal integral of the zero-mean gas load fluctuations. Results for exemplary cases show that the 255 sensitivity of pressure fluctuations to gas load fluctuations is peaked at and around locations of stationary mass flux reversals. The 256 results suggest the development of a risk-aware gas compressor control that limits the probability of the gas pressure exceeding 257 upper engineering limits or lower contract delivery limits. Pipelines with spatially-disordered injections and loads show less 258 sensitivity to gas load fluctuations.

259 There are many areas for future work including:

260 - The current formulation should be converted to a node-edge network model more amenable to the simulation of real gas networks with compression concentrated at gas compressor stations.

262

- Discrete compressor stations will force the relaxation of our assumption of spatially uniform pressure.

- The solution for the stationary compression should be converted to an optimization for gas pipeline operations (e.g. for minimum cost of compression, maximum throughput, etc) while limiting the probability of violating an upper or lower gas pressure limit.

267 The authors acknowledge multiple discussions with R. Bent and S. Misra, and A. Zlotnik for discussions and help with 268 references. The work at LANL was carried out under the auspices of the National Nuclear Security Administration of the 269 U.S. Department of Energy at Los Alamos National Laboratory under Contract No. DE-AC52-06NA25396. MC and SB also 
270 acknowledge partial support of the Advanced Grid Modeling Program in the US Department of Energy Office of Electricity, 271 DTRA Basic Research Project \#10027-13399 and the NSF/ECCS collaborative research project on Power Grid Spectroscopy 272 through NMC.

274 The Supplementary Information contained in the Appendixes describes in greater detail the physical models of gas flow and 275 the approximations used to develop the models discussed in the main text.

276 Methods used to derive main results of the paper are detailed in Appendix B, consisting of two Subsections devoted to discus277 sion of the linearized one-dimensional model of stochastic gas dynamics and following analysis of the probabilistic measure of 278 risk, respectively.

279 The remaining Appendixes provide discussions of more general modeling needed to support the paper's conclusions. Ap280 pendix $\mathrm{C}$ describes basic hydrodynamic equations for a single pipe and discusses the slow transient approximation used in the 281 main text. Appendix D generalizes the single pipe Dynamic Gas Flow (DGF) description to the case of non-steady gas flows 282 over a meshed network. Appendix E briefly discusses steady Gas Flow (GF) solutions of the DGF model and puts them in the 283 context of the Optimum Gas Flow (OGF) problem used to determine gas compressor operation. In Appendix F, the DGF system 284 is linearized around a steady solution and the general solution of the linear dynamic problem over the network is constructed. 285 The solution is split into homogeneous (zero mode) and inhomogeneous parts and it is argued that the inhomogeneous part of the 286 linearized DGF becomes asymptotically small in the regime of interest. Here we also add a Subsection briefly discussing the in287 homogeneous correction, for the general case and then also for the special model of a long pipeline with distributed compression 288 discussed in the main text.

\section{Linearized Model of Stochastic Gas Dynamics}

291 The stationary solution described above applies to gas pipelines with well-behaved gas loads. Under these conditions, the 292 pressure $p_{0}$ does not vary and the pipeline operations are very secure and reliable. Stochastic gas loads that arise from gas 293 generation compensating fluctuating renewable generation change this picture. Fluctuating gas loads are added to the stationary 294 solution

$$
q^{(\mathrm{st})}(x) \rightarrow q(t ; x)=q^{(\mathrm{st})}(x)+\xi(t ; x),
$$

295 where $\xi(t ; x)$ is zero mean $(\langle\xi\rangle=0)$ so that each load, although stochastic, is restricted to consume its scheduled amount $q^{(\text {st })}(x)$ 296 over the intra-day market subperiod. The stochastic component of the gas load $\xi(t ; x)$ is expected to include spatiotemporal 297 correlations typical of renewable generation, e.g. $\xi(t ; x)$ for wind generation is expected to be correlated on the time scale of 298 tens of minutes to hours over lengths from tens to hundreds of miles.

299 The effect of the stochastic gas loads is analyzed by linearizing the hydrodynamic model in Eqs. (1,2). (Linearization of 300 the basic non-stationary gas flow equations was already discussed in the literature, however only in the context of simplifying 301 numerical evaluations of the underlying partial differential equations (e.g. ${ }^{38}$ and references therein). Here, we carry it two steps 302 further-we derive analytical relations and then to analyze effects of stochastic fluctuations and spatial disorder in gas loads. 303 Another recent analytical approach retains the basic nonlinearity but assumes adiabaticity, i.e. very slow changes in the gas 304 loads $^{39}$. Although promising computationally, this approach fails to account for fast, but not necessarily large, fluctuations in 305 the gas draws originating from the electric grid-natural gas pipeline interaction.) Expanding these equations to first order in the 306 fluctuations yields

$$
\begin{aligned}
c_{s}^{-2} \partial_{t} \delta p+\partial_{x} \delta \phi & =-\xi \\
\partial_{x} \delta p+\frac{\beta}{d} \frac{\phi^{(\mathrm{st})} \delta \phi}{p_{0}}-\frac{\beta}{d} \frac{\left(\phi^{(\mathrm{st})}\right)^{2}}{p_{0}^{2}} \delta p & =0
\end{aligned}
$$

307 where $\delta p$ and $\delta \phi$ are the fluctuating pipeline pressures and mass flows, respectively. Although the gas loads fluctuate, the gas 308 injections $\phi_{0}$ and $\phi_{L}$ remain at their stationary values imposing conditions on the fluctuating mass flows

$$
\int_{0}^{L} d x \partial_{x} \delta \phi=0 .
$$

309 The structure of Eqs. (B2-B4) provides some guidance regarding the types of solutions expected. Differentiating Eq. (B3) 310 with respect to $x$ (and temporarily assuming a uniform $\phi^{(\mathrm{st})}$ ) enables the elimination of $\delta \phi$ via Eq. (B2). The resulting PDE in 
$311 \delta p$ has the structure of a nonlinear diffusion equation that is driven by exogenous perturbations $\xi(t ; x)$. Because $\langle\xi\rangle=0$ over the 312 intra-day periods, it is tempting to drop all time derivatives in Eq. (B4) and compute time-independent mean square fluctuations 313 of $\delta p$, however, this approach is incomplete. Spatiotemporal correlations in $\xi(t ; x)$ occurring on time scales shorter than the 314 intra-day period will result in gas draw fluctuations that create shorter-term imbalance with net flow of gas into or out of the 315 pipeline. Eq. (B4) shows that these non-zero net fluctuations are not allowed to leak out either end of the pipeline because $\phi_{0}$ 316 and $\phi_{L}$ are held fixed. The only way for the system to accommodate these short-term correlated fluctuations is through a "zero 317 mode" where the average pressure in the entire pipeline raises or falls along with the fluctuating injections. This zero mode and 318 its effects on the pipeline pressure fluctuations are the emphasis of the remainder of this manuscript.

\section{Probabilistic Measure of Risk: Analysis}

320

To represent the slow drifts of pipeline pressure discussed above, we suggest a solution to Eqs. (B2,B3) of the form

$$
\delta p(t ; x)=a(t) Z(x)+b(t ; x),
$$

321 where the two components of the solution respond to the different characteristics of the fluctuations $\xi(t ; x)$. The first component $322 a(t) Z(x)$ is the zero mode where $Z(x)$ is a slowly varying function of $x$ that captures the spatial distribution of gas stored in the ${ }_{323}$ pipeline as pressure rise or fall driven by the correlated component of the fluctuating gas draws. The time dependence of these 324 global pressure swings are captured by $a(t)$. In contrast, $b(t ; x)$ varies more rapidly in space and responds to the uncorrelated 325 component of the fluctuations of $\xi(t ; x)$ that occur on finer spatial and time scales. The larger spatial derivatives of $b(t ; x)$ result 326 in relatively rapid diffusion of pressure (and gas) which limits the impact of $b(t ; x)$ on pressure fluctuations.

327 Substituting our proposed solution (B5) into Eqs. (B2,B3) yields an equation for the zero mode

$$
\partial_{x} Z-\frac{\beta}{d} \frac{\phi^{(\mathrm{st})}\left(x^{\prime}\right)\left|\phi^{(\mathrm{st})}\left(x^{\prime}\right)\right|}{p_{0}^{2}} Z=0,
$$

328 which has a solution

$$
Z(x)=\exp \left[\int_{0}^{x} d x^{\prime} \frac{\beta \phi^{(\mathrm{st})}\left|\phi^{(\mathrm{st})}\right|}{d p_{0}^{2}}\right]=\exp \left[\int_{0}^{x} 2 \gamma\left(x^{\prime}\right) d x^{\prime}\right] .
$$

${ }_{329}$ The solution for $Z(x)$ does not depend on the form of the fluctuations $\xi$. Rather, it depends on the stationary solution $\phi^{(\text {st })}(x)$, 330 or equivalently on deployed gas compression in the stationary solution. The same substitution also yields an expression for $a(t)$ 331 that does depend on the gas load fluctuations:

$$
c_{s}^{-2} Z \partial_{t} a+\delta_{x} \delta \phi=-\xi
$$

332 Since $a(t)$ is independent of $x$, Eq. (B8) can be integrated over the length of the pipeline to yield an explicit expression for $a(t)$ :

$$
a=-\frac{c_{s}^{2}}{L Y} \int_{0}^{t} d t^{\prime} \int_{0}^{L} d x \xi\left(t^{\prime}, x\right), \quad Y=\int_{0}^{L} d x Z / L,
$$

333 where Eq. (B4) has been used to eliminate the $\delta \phi$ term.

${ }_{334}$ The physical interpretation of the zero mode $a(t) Z(x)$ now becomes clear. The double integral in Eq. (B9) filters out the 335 uncorrelated components of $\xi$ showing that the time dependence of the zero mode $a(t)$ only responds to the fluctuations of $\xi$ that ${ }_{336}$ are correlated in space (over the entire length of the pipeline) and in time (since the beginning of the intra-day market period). 337 A discussion of the solution component $b(t ; x)$ is given below in Section $\mathrm{F} 1$.

${ }_{338}$ The zero mode $a(t) Z(x)$ will dominate the contribution to $\delta p(t)$ at times longer than the correlation time $\tau$ of $\xi$ where $\tau$ 339 is expected to be in the range of tens of minutes to hours for fluctuating gas loads creating by gas-fired electric generators 340 balancing intermittent wind generation. For $t \gg \tau$, we may safely drop the $b$ in favor of $a(t) Z(x)$ and estimate the pressure 341 variation covariance as

$$
\left\langle(\delta p(x))^{2}\right\rangle=\frac{c_{s}^{4} \tau t}{L^{2}}\left(\frac{Z(x)}{Y}\right)^{2} \iint_{0}^{L} d x_{1} d x_{2}\left\langle\xi\left(t, x_{1}\right) \xi\left(t, x_{2}\right)\right\rangle,
$$

342 where we have also assumed statistical stationarity of $\xi(t ; x)$ over time.

${ }_{343}$ Eq. (B10) can be rearranged slightly to reveal a physical interpretation, as shown in Fig. (6) of the main text. The first term on 344 the right hand side of Eq. (6) of the main text is the square of the fractional pressure decline if the entire pipeline was subject to 345 the spatially averaged gas load $\bar{q}^{(\mathrm{st})}$ without any compensating injections for one correlation time $\tau$ of the gas load fluctuations. 
346 This first term is multiplied by the number of correlation times $(t / \tau)$ since the intra-day period began. The third term provides the ${ }_{347}$ only $x$ dependence and describes the sensitivity of different locations in the pipeline to pressure fluctuations. This dependence 348 comes entirely through $Z(x)$ which (see Eq. (B7)) depends only on the compression deployed in the stationary solution. The 349 dependence on $Z(x)$ demonstrates that a highly stressed pipeline, i.e. one with a large $\int \gamma\left(x^{\prime}\right) d x^{\prime}$, is more susceptible to pressure 350 fluctuations driven by stochastic gas loads, and $Z(x)$ shows which pipeline locations are most susceptible. The final term on 351 the the right hand side of Eq. (6) of the main text measures the spatial average of the correlated fluctuations in the gas loads 352 normalized by the average stationary gas load.

${ }_{353}$ The right hand side of expression for $a(t)$ in Eq. (B9) is a time integral over a stochastic process, and per the law of large 354 numbers, $a(t ; x)$ and $\delta p(t ; x)$ are expected to be asymptotically Gaussian when the integration time is longer than the correlation 355 time of $\xi$. In this limit, the estimate of the pressure fluctuation covariance in Eq. (B10) or Eq. (6) of the main text also predicts the 356 tails of the distribution over $\delta p$, thus allowing the estimation of the probability of relatively rare events of high or low pressure 357 fluctuations (under the condition that the fluctuations are still within the linear approximation used here). Eq. (B10) becomes a ${ }_{358}$ probabilistic measure of risk to reliability of natural gas pipeline operations and a route to modeling the risk that cascades to the 359 interdependent infrastructures such as electric power systems.

\section{Appendix C: Gas Flow Equations: Individual Pipe}

${ }_{361}$ Following $^{36}$, we consider the flow of a compressible gas in a single length of pipe. Major transmission pipelines are typically 362 16-48 inches in diameter and operate at high pressures and mass flows, e.g. 200 to 1500 pounds per square inch (psi) and moving 363 millions of cubic feet of gas per day ${ }^{40,41}$. Under these highly turbulent conditions, the pressure drop and energy loss due to shear 364 is well represented by a nearly constant phenomenological friction factor $f$. The resulting gas flow model is a partial differential 365 equation (PDE) with one spatial dimension $x$ (along the pipe axis) and one time dimension ${ }^{7-9}$ :

$$
\begin{aligned}
& \partial_{t} \rho+\partial_{x}(u \rho)=0, \\
& \partial_{t}(\rho u)+\partial_{x}\left(\rho u^{2}\right)+\partial_{x} p=-\frac{\rho u|u|}{2 d} f-\rho g \sin \alpha, \\
& p=\rho Z R T .
\end{aligned}
$$

${ }_{366}$ Here, $u, p, \rho$ are velocity, pressure, and density at position $x ; Z$ is the gas compressibility factor; $T$ is the temperature, $R$ is the 367 gas constant, and $d$ is the diameter of the pipe.

368 Eqs. $(\mathrm{C} 1, \mathrm{C} 2, \mathrm{C} 3)$ represent mass conservation, momentum balance and the ideal gas thermodynamic relation, respectively. 369 The first term on the rhs of Eq. (C2) represents the friction losses in the pipe. The second term on the rhs of Eq. (C2) accounts 370 for the gain or loss of momentum due to gravity $g$ if the pipe is tilted by angle $\alpha$. The frictional losses typically dominate the 371 gravitational term, which is typically dropped. Similarly, the gas inertia term $\left(\partial_{t}(\rho u)\right.$ is also typically small compared to the 372 frictional losses (as the flow velocity is significantly smaller than sound velocity) and is dropped. For simplicity of presentation, 373 we have also assumed that the temperature does not change significantly along the pipe.

374 Taking into account these assumptions, Eqs. $(\mathrm{C} 1, \mathrm{C} 2, \mathrm{C} 3)$ are rewritten in terms of the pressure $p$ and the mass flux $\phi=u \rho$ :

$$
\begin{aligned}
& c_{s}^{-2} \partial_{t} p+\partial_{x} \phi=0, \\
& \partial_{x} p+\frac{\beta}{2 d} \frac{\phi|\phi|}{p}=0,
\end{aligned}
$$

375 where $c_{s} \equiv \sqrt{Z R T}$ is the sound velocity and $\beta \equiv f Z R T$ are both considered constant. To resolve the dynamic problem for ${ }_{376} t \in[0, \tau]$ over $x \in[0, L]$ we also need to supply Eqs. (C4,C5) with initial and boundary conditions for flows,

$$
\begin{aligned}
& t=0, \quad \forall x \in[0, L]: \quad \phi(0 ; x)=\phi_{0}(x), \\
& \forall t: \quad \phi(t ; 0)=q^{(\text {in })}(t), \quad \phi(t ; L)=q^{(\text {out })}(t),
\end{aligned}
$$

377 which are consistent, i.e. $\phi_{0}(0)=q^{(\mathrm{in})}(0)$ and $\phi_{0}(L)=q^{(\mathrm{out})}(0)$, in addition fixing pressure initially at an end of the pipe, e.g. ${ }_{378} p(0 ; 0)=p_{0}$.

${ }_{380}$ The single pipe setting in Eqs. $\mathrm{C} 4$ and $\mathrm{C} 5$ is generalized to a gas network represented by a graph $\mathcal{G}=(\mathcal{V}, \mathcal{E})$ with a set of ${ }_{381}$ vertexes $\mathcal{V}$ and set of edges $\mathcal{E}$, where the edges will be considered directed or undirected, depending on the context. See Fig. 8 382 for a schematic illustration. We will adopt $(i, j)$ and $\{i, j\}$ notations for directed and undirected edges, respectively. Each vertex, 
a)

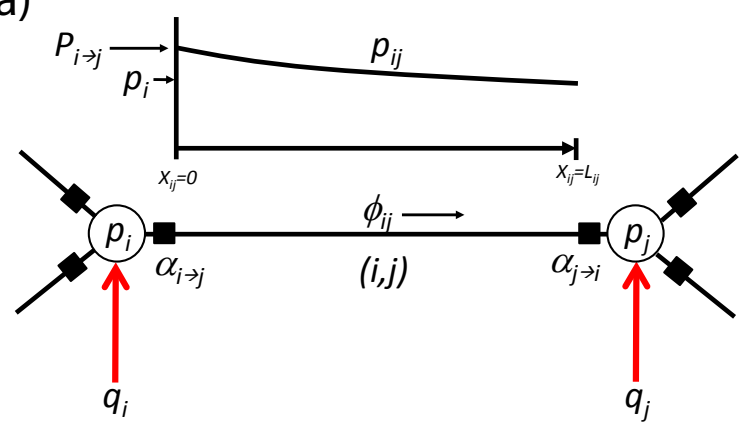

b)

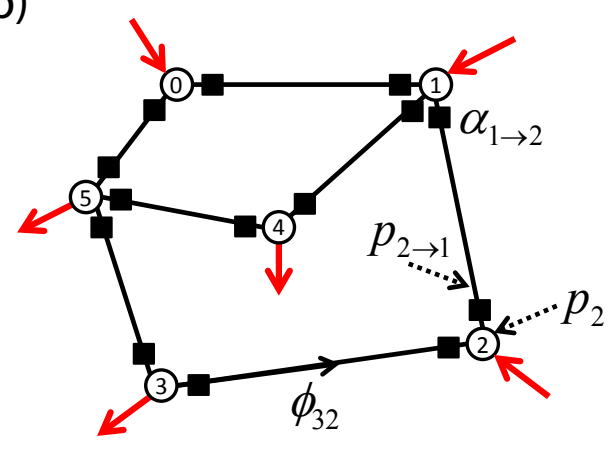

FIG. 8. Schematic illustration of the network-structure notations. a) Schematic illustration of a single edge $(i, j)$ of a network. Nodes at either end are indicated by open circles and labeled by their nodal pressure $p_{i}$ and $p_{j}$. Compressors are indicated with filled squares. Mass flow $\phi_{i j}$ is directed from $i$ to $j$ and injections $q_{i}$ and $q_{j}$ contribute to this flow. Nodal pressure $p_{i}$ is modified by the compression ratio $\alpha_{i \rightarrow j}$ yielding $p_{i j}\left(x_{i j}=0\right)$. The pressure falls along $\{i, j\}$ reaching $p_{i j}\left(x_{i j}=L_{i j}\right)$. If compressor $\alpha_{j \rightarrow i}$ is not present, then $p_{i j}\left(x_{i j}=L_{i j}\right)=p_{j}$. b) Schematic of many edges connected in a meshed network. Nodes are indexed by $i=0,1, \cdots$, where node 0 is typically reserved for the swing bus - the node where pressure is maintained constant throughout the dynamics. Compressors and injections and edge mass flows are the same as in a).

${ }_{383} i \in \mathcal{V}$ represents a node with a gas injection/consumption rate $q_{i}$ (mass per unit time). Each edge $(i, j) \in \mathcal{E}$ is a single pipe with 384 mass flow $\phi_{i j}$. The flow along each edge is described by a set of PDEs:

$$
\begin{aligned}
& \forall t \in[0, \tau], \quad \forall\{i, j\} \in \mathcal{E}, \quad \forall x \in\left[0 ; L_{i j}\right]: \\
& c_{s}^{-2} \partial_{t} p_{i j}(t, x)+\partial_{x} \phi_{i j}(t, x)=0, \\
& \partial_{x} p_{i j}(t, x)+\frac{\beta}{2 d} \frac{\phi_{i j}(t, x)\left|\phi_{i j}(t, x)\right|}{p_{i j}(t, x)}=0,
\end{aligned}
$$

385 where $p_{i j}(t, x)$ and $\phi_{i j}(t, x)$ are the pressure and mass flow, respectively, at time $t$ and position $x$ along edge $(i, j)$ of length ${ }_{386} L_{i j}$. Here, $p_{i j}=p_{j i}, \phi_{i j}=-\phi_{j i}$, and $L_{i j}=L_{j i}$. See Fig. 8a for a schematic description of the variables.

387 The flow of gas create a pressure drop. To compensate, the pressure is boosted at compressor stations potentially located at 388 both ends of each edge $\{i, j\} . \alpha_{i \rightarrow j}$ is the compression ratio of the station adjacent to node $i$ while $\alpha_{j \rightarrow i}$ is the compression ratio 389 adjacent to node $j$. We choose to place compressors at the two ends of every line/pipe for generality, which also simplifies the 390 notations in the following discussion. In reality there will be only none or one compressor on any particular edge of the graph. ${ }_{391}$ Note also that $\alpha_{i \rightarrow j}$ may be larger or smaller than unity, thus representing compression or decompression. If only compression 392 is allowed, then $\alpha_{i \rightarrow j} \geq 1$. The relationships between the pressures in Fig. 8 are

$$
\begin{aligned}
& \forall t \in[0, \tau], \quad \forall(i, j) \in \mathcal{E}: \quad p_{i j}(t, 0)=p_{i \rightarrow j}(t), \\
& p_{i j}\left(t, L_{i j}\right)=p_{j \rightarrow i}(t), p_{i \rightarrow j}=p_{i} \alpha_{i \rightarrow j}, p_{j \rightarrow i}=p_{j} \alpha_{j \rightarrow i},
\end{aligned}
$$

393 where $p_{i}$ and $p_{i \rightarrow j}$ are the pressures at node $i$ and just past the compressor on edge $(i, j)$ adjacent to node $i$ and the last part $394 \mathrm{of} \mathrm{Eq.} \mathrm{(D3)} \mathrm{is} \mathrm{added} \mathrm{for} \mathrm{clarity.} \mathrm{If} \mathrm{there} \mathrm{is} \mathrm{no} \mathrm{compressor} \mathrm{installed} \mathrm{at} \mathrm{the} \mathrm{beginning} \mathrm{of} \mathrm{the} \mathrm{edge}(i, j)$ or if the compressor 395 is inactive, $\alpha_{i \rightarrow j}=1$. In the current operational paradigm, compression rates are not changed very frequently, however, we 396 anticipate changes and allow the $\alpha_{i \rightarrow j}$ to depend on time.

397 Eqs. (D2,D3) are complemented with mass conservation at all nodes of the network:

$$
\forall t \in[0, \tau], \quad \forall i \in \mathcal{V}: \sum_{j:(i, j) \in \mathcal{E}} \phi_{i j}(t, 0)=q_{i}(t) .
$$

398 When the gas injections $q(t)=\left(q_{i}(t) \mid i \in \mathcal{V}\right)$ for are given for $t \in[0, \tau]$, nodal conditions (D4) generalize the single-pipe 399 boundary conditions in (C7) to a pipe network. Eqs. (D1,D2,D3,D4) constitute a complete set of equations describing the 400 Dynamic Gas Flow (DGF) problem if they are supplemented with compression ratios, i.e. $\alpha=\left(\alpha_{i \rightarrow j} \mid(i, j) \in \mathcal{E}\right)$, initial 401 conditions on the flows

$$
\begin{aligned}
& t=0, \forall\{i, j\} \in \mathcal{E}, \forall x_{i j} \in\left[0, L_{i j}\right]: \\
& \phi_{i j}\left(0 ; x_{i j}\right)=\phi_{i j}^{(i n)}\left(x_{i j}\right),
\end{aligned}
$$

402 and pressure at one arbitrarily chosen slack node, $p_{i=0}(0)=p_{0}$. 
404 The stationary/steady version of the DGF problem is the Gas Flow (GF) problem. In the GF problem, all input parameters 405 (consumptions/injections, compression ratios and the pressure at the slack bus) are constant in time, and the total injection and 406 consumption are balanced

$$
\sum_{i \in \mathcal{V}} q_{i}^{(\mathrm{st})}=0
$$

407 The steady solution of Eq. (D1) is uniform mass flow along each pipe in the network, $\forall\{i, j\}: \quad \phi_{i \rightarrow j}=$ const. Substituting this 408 result into Eq. (D2) and taking straightforward spatial integration yields algebraic relations between flow through and pressures 409 at both ends of every pipe in the network

$$
\forall(i, j) \in \mathcal{E}: \quad p_{i \rightarrow j}^{(\mathrm{st})}=p_{i}^{(\mathrm{st})} \alpha_{i \rightarrow j} ; \quad\left(p_{i j}^{(\mathrm{st})}(x)\right)^{2}=\left(p_{i \rightarrow j}^{(\mathrm{st})}\right)^{2}-\frac{\beta x}{d} \phi_{i j}^{(\mathrm{st})}\left|\phi_{i j}^{(\mathrm{st})}\right| .
$$

410 The GF problem has a unique solution provided the compression ratios are known.

411 Compression ratios $\alpha$ are time-independent in the steady GF setting. The configuration of $\alpha$ over the network is typically 412 decided using a combination of economic and operational factors. The model selected in the main text corresponds to a simple 413 greedy approach, i.e. maintain constant pressure throughout the network for flows corresponding to the forecasted comsump414 tions/injections. This model roughly replicates the behavior of pipeline operators in the U.S. where the energy consumed in the 415 compression of the gas is not a major concern. More sophisticated compression dispatch options, in particular minimization of 416 the total work spent on compression subject to maintaining pressure within acceptable limits, have been extensively discussed 417 in the literature, e.g. ${ }^{33,34,36,42}$ and references therein.

\section{Appendix F: Perturbative solution of the DGF problem}

419 We generalize discussion in the main text by introducing stochastic gas loads (due, e.g., to natural gas-fired generators) from 420 a line to a network, such that $q(t)=q^{(\mathrm{st})}+\xi(t)$ where components of $\xi(t)=\left(\xi_{i}(t) \mid i \in \mathcal{V}\right)$ are time varying but relatively 421 small in comparison with $q^{(\mathrm{st})}$. We look for a linearized solution of the DGF problem of the form $p(t)=p^{(\mathrm{st})}+\delta p(t)$ and ${ }_{422} \phi(t)=\phi^{(\mathrm{st})}+\delta \phi(t)$, where the respective corrections are small, i.e. $|\delta p(t)| \ll p^{(\mathrm{st})}$ and $|\delta \phi(t)| \ll \phi^{(\mathrm{st})}$. The linearized 423 versions of Eqs. (D1,D2,D3,D4) become

$$
\begin{aligned}
& \forall t \in[0, \tau], \quad \forall\{i, j\} \in \mathcal{E}, \quad \forall x \in\left[0 ; L_{i j}\right]: \\
& \quad c_{s}^{-2} \partial_{t} \delta p_{i j}+\partial_{x} \delta \phi_{i j}=0, \\
& \partial_{x} \delta p_{i j}+\frac{\beta}{2 d}\left(\frac{\delta \phi_{i j}\left|\phi_{i j}^{(\mathrm{st})}\right|}{p_{i j}^{(\mathrm{st})}}+\frac{\phi_{i j}^{(\mathrm{st})}\left|\delta \phi_{i j}\right|}{p_{i j}^{(\mathrm{st})}}-\frac{\delta p_{i j} \phi_{i j}^{(\mathrm{st})}\left|\phi_{i j}^{(\mathrm{st})}\right|}{\left(p_{i j}^{(\mathrm{st})}\right)^{2}}\right)=0, \\
& \forall t \in[0, \tau], \quad \forall(i, j) \in \mathcal{E}: \quad \delta p_{i \rightarrow j}=\delta p_{i} \alpha_{i \rightarrow j}, \\
& \quad \delta p_{i j}(t, 0)=\delta p_{i \rightarrow j}(t), \quad \delta p_{i j}\left(t, L_{i j}\right)=\delta p_{j \rightarrow i}(t), \\
& \forall t \in[0, \tau], \quad \forall i \in \mathcal{V}: \quad \sum_{j:(i, j) \in \mathcal{E}} \delta \phi_{i j}(t, 0)=\xi_{i}(t) .
\end{aligned}
$$

424 The remainder of the Subsection is devoted to finding an asymptotic solution of Eqs. (F1,F2,F3,F4,F5). Here, asymptotic 425 implies finding solutions for time $\tau$ longer than the correlation time of the fluctuation consumption $\xi$. We seek solutions that 426 eliminate the complexity of the PDE of Eqs. (F1, F2,F3,F4,F5) and that connect the nodal quantities by algebraic relationships. ${ }_{427}$ Therefore, generalizing the solution proposed in the main text (see Eq. (10)), we look for a solution of Eqs. (F1,F2) of the 428 form

$$
\delta p_{i j}=a_{i j}(t) Z_{i j}(x)+b_{i j}(t, x)
$$

${ }_{429}$ where $a_{i j}(t)$ only depends on time. Here in Eq. (F6) $Z_{i j}(x)$ solves the following linear homogeneous equation

$$
\partial_{x} Z_{i j}-\frac{\beta}{d} \frac{\phi_{i j}^{(\mathrm{st})}\left|\phi_{i j}^{(\mathrm{st})}\right|}{p_{i j}^{(\mathrm{st})}} Z_{i j}=0
$$

${ }_{430}$ where $Z_{i j}(x)$ counts $x$ from node $i$, i.e. reversing the direction of counting one gets, $Z_{i j}\left(L_{i j}\right)=Z_{j i}(0)$. 
${ }_{431}$ Assuming that $\tau$ is sufficiently large, we conjecture (which will be verified after the global asymptotic solution is found) that 432 the major contribution to $\delta p_{i j}$ in Eq. (F6) originates from the first "zero-mode" term $a_{i j}(t) Z_{i j}(x)$ that (as will be seen below) ${ }_{433}$ grows in time compared to the second term that does not.

${ }_{434}$ To find the leading (zero mode) term we proceed as follows. The integration of Eq. (F7) over the spatial dependence of the 435 stationary profile (E2), yields

$$
Z_{i j}(x)=\frac{p_{i \rightarrow j}^{(\mathrm{st})}+p_{j \rightarrow i}^{(\mathrm{st})}}{2 p_{i j}^{(\mathrm{st})}(x)},
$$

${ }_{436}$ where the normalization constant is chosen to guarantee, $\int_{0}^{L} Z_{i j}(x) d x / L=1$. We solve for the time-dependent factor $a_{i j}(t)$ by ${ }_{437}$ substituting $\delta p_{i j}$ with $a_{i j}(t) Z_{i j}(x)$ into Eq. (F1) and integrate the result over the entire spatial extent of the pipe $\{i, j\}$ yielding

$$
a_{i j}(t)=c_{s}^{2} \int_{0}^{t} d t^{\prime}\left(\delta \phi_{i j}\left(t^{\prime}, 0\right)-\delta \phi_{i j}\left(t^{\prime}, L\right)\right) .
$$

${ }_{438}$ In the asymptotic limit where $\delta p_{i j}$ is approximated by $a_{i j}(t) Z_{i j}(x)$ for every pipe (graph edge), Eqs. (F4) can only be satisfied ${ }_{439}$ if the $a_{i j}(t)$ have the same functional dependence on time, i.e.,

$$
\forall\{i, j\} \in \mathcal{E}: \quad a_{i j}(t)=a(t) c_{i j},
$$

${ }_{440}$ where $c_{i j}=c_{j i}$ is an edge specific constant. To compute the global time-dependent factor $a(t)$ in Eq. (F10) we sum over all the 441 nodes of the graph

$$
\sum_{i \in \mathcal{V}} \xi_{i}=\sum_{\{i, j\} \in \mathcal{E}}\left(\delta \phi_{i j}(t, 0)-\delta \phi_{i j}\left(t, L_{i j}\right)\right)
$$

442 integrate over time, define

$$
\Xi(t) \doteq \int_{0}^{t} d t^{\prime} \sum_{i \in \mathcal{V}} \xi_{i}\left(t^{\prime}\right)
$$

${ }_{443}$ and finally sum Eq. (F10) overall edges:

$$
a(t)=\frac{c_{s}^{2} \Xi(t)}{\sum_{\{i, j\} \in \mathcal{E}} c_{i j}} .
$$

${ }_{444}$ Therefore, $\forall t, \forall\{i, j\} \in \mathcal{E}, \quad x \in\left[0, L_{i j}\right]$ :

$$
\delta p_{i j}(t, x) \approx \frac{c_{s}^{2} \Xi(t)}{\sum_{\{i, j\} \in \mathcal{E}} c_{i j}} c_{i j} Z_{i j}(x) .
$$

${ }_{445}$ The unknown edge constants $c_{i j}$ are derived by substituting Eqs. (F14) into Eqs. (F3, F10) yielding

$$
\forall i, \forall j, k \text { s.t. }(i, j),(i, k) \in \mathcal{E}: \frac{c_{i j} Z_{i j}(0)}{\alpha_{i \rightarrow j}}=\frac{c_{i k} Z_{i k}(0)}{\alpha_{i \rightarrow k}} .
$$

${ }_{446}$ Eqs. (F14, F15, F8) express the complete asymptotic (zero mode) solution of the DGF problem.

${ }_{447}$ Assuming that the random gas load fluctuations $\xi_{i}(t)$ are zero-mean, temporarily homogeneous, and relatively short correlated 448 in both time (the correlation time is less than $\tau$ ) and space (the correlation length is less than the spatial extent of the network), 449 and observing that $\delta p_{i j}$ in Eq. (F14) is given by a time-integral and spatial-sum of the fluctuations, one concludes that according 450 to the Large Deviation theory, the pressure fluctuations form a Gaussian random process which jitter diffusively in time, i.e. the ${ }_{451}$ Probability Distribution Function (PDF) of $\delta p_{i j}(t, x)$ is

$$
\begin{aligned}
& \mathcal{P}\left(\delta p_{i j}(t, x)=\delta\right) \rightarrow\left(2 \pi t D_{i j}(x)\right)^{-1 / 2} \exp \left(-\frac{\delta^{2}}{2 t D_{i j}(x)}\right), \\
& D_{i j}=\left(\frac{c_{s}^{2} c_{i j} Z_{i j}(x)}{\sum_{\{k, l\} \in \mathcal{E}} c_{k l}}\right)^{2}\left\langle\left(\sum_{n \in \mathcal{V}} \xi_{n}\left(t^{\prime}\right)\right)^{2}\right\rangle,
\end{aligned}
$$

${ }_{452}$ where the correlation function on the right-hand-side does not depend on $t^{\prime}$ due to assumption of the statistical homogeneity of $453 \xi$. 
455 In the general analysis of the preceding Section of this SI, the pressure fluctuations are separated into homogeneous (zero 456 mode) and inhomogeneous (forced) components, according to Eq. (F6). The formal separation in Eq. (F6) leads to a differential ${ }_{457}$ equation for the inhomogenous solution $b(t ; x)$ which, for the general formulation above, is fully defined by Eqs. (F18,F19).

${ }_{458}$ Once the leading, growing in time, contribution to $\delta p_{i j}$ is found, one verifies that, $b_{i j}(t, x)$, extracted from

$$
\begin{aligned}
& \partial_{x} b_{i j}+\frac{\beta}{2 d}\left(\frac{\delta \phi_{i j}\left|\phi_{i j}^{(\mathrm{st})}\right|}{p_{i j}^{(\mathrm{st})}}+\frac{\phi_{i j}^{(\mathrm{st})}\left|\delta \phi_{i j}\right|}{p_{i j}^{(\mathrm{st})}}-\frac{\delta p_{i j} \phi_{i j}^{(\mathrm{st})}\left|\phi_{i j}^{(\mathrm{st})}\right|}{\left(p_{i j}^{(\mathrm{st})}\right)^{2}}\right)=0 . \\
& c_{s}^{-2} Z_{i j} \frac{d}{d t} a_{i j}+\partial_{x} \delta \phi_{i j}=0,
\end{aligned}
$$

${ }_{459}$ does not grow with time, and thus it is asymptotically smaller — consistently with what was conjectured above to derive the 460 leading contribution.

${ }_{461}$ Let us discuss this asymptotic separation of the solution into dominant contribution and correction in more details for the 462 simplified analysis/model of the main text. Repeating the solution separation on the (simplified) continuous-compression model, 463 we find a differential equation for $b(t ; x)$ in terms of $\delta \phi$, i.e.

$$
\partial_{x} b+\frac{\beta}{d} \frac{\left|\phi^{\mathrm{st}}\right| \delta \phi+\phi^{\mathrm{st}}|\delta \phi|}{2 p_{0}}-\frac{\beta}{d} \frac{\left(\phi^{\mathrm{st}}\right)^{2}}{p_{0}^{2}} b=0 .
$$

${ }_{464}$ Fluctuations in $\delta \phi$ drive $b(t ; x)$, but unlike for the homogeneous solution, Eq. (F20) shows that this effect is entirely local. 465 Specifically, Eq. (14) of the main text shows that the homogeneous component responds to the global imbalance in gas loads 466 while the response in Eq. (F20) is to the local $\delta \phi$. In addition, the response in Eq. (F20) decays in space and does so quickly in 467 areas of high compression for the stationary solution (see Eq. (5) of the main text). In contrast, the homogeneous solution grows 468 more quickly in areas of high compression (see Eq. (12) of the main text). These two properties contribute to the dominance of 469 the homogeneous solution over the inhomogeneous solution at longer times.

$47{ }^{1}$ An overview of modern shale gas development in the united states, http://www.all-llc.com/publicdownloads/ 471 ALLShaleOverviewF INAL.pdf, 2008.

$472{ }^{2}$ ISO New England: Adressing Gas Dependence, http://www.iso-ne.com/committees/comm_wkgrps/strategic_ 473 planning_discussion/materials/natural-gas-white-paper-draft-july-2012 . pdf, 2012.

474 Levelized cost of electricity renewable energy technologies, http://www.ise.fraunhofer.de/ 475 en/publications/veroeffentlichungen-pdf-dateien-en/studien-und-konzeptpapiere/

476 study-levelized-cost-of-electricity-renewable-energies.pdf, 2013.

$477{ }^{4}$ Renewable portfolio standards in the states: Balancing goals and implementation strategies, http://www. nrel.gov/docs/ 478 fy08osti/41409.pdf, 2007.

4795 The Future of Natural Gas:MIT Energy Initiative, http://mitei.mit.edu/system/files/NaturalGas_Report.pdf, 2010.

$480{ }^{6}$ Growing concerns, possible solutions: The interdependency of natural gas and electricity systems, http://mitei.mit.edu/ 481 system/files/2014-MITEI-Report-Growing-Concerns-Possible-Solutions.pdf, 2014.

4827 A. Osiadacz. Simulation and analysis of gas networks. Gulf Pub. Co., 1987.

${ }_{483}{ }^{8}$ A.R.D. Thorley and C.H. Tiley. Unsteady and transient flow of compressible fluids in pipelinesa review of theoretical and some experimental 484 studies. International Journal of Heat and Fluid Flow, 8(1):3 - 15, 1987.

4859 S. A. Sardanashvili. Computational Techniques and Algorithms (Pipeline Gas Transmission) [in Russian]. FSUE Oil and Gaz, I.M. Gubkin, 486 Russian State University of Oil and Gas, 2005.

${ }_{487}{ }^{10}$ M. K. Banda, M. Herty, and A. Klar. Gas flow in pipeline networks. Networks and Heterogeneous Media, 1:41-56, 2006.

${ }_{488}{ }^{11}$ M. K. Banda and M. Herty. Multiscale modeling for gas flow in pipe networks. Mathematical Methods in the Applied Sciences, 31:915-936, 4892008.

$490{ }^{12}$ Seungwon An, Qing Li, and Thomas W Gedra. Natural gas and electricity optimal power flow. In Transmission and Distribution Conference 491 and Exposition, 2003 IEEE PES, volume 1, pages 138-143. IEEE, 2003.

${ }_{492}{ }^{13}$ C. Unsihuay, J. W. Marangon-Lima, and A. C. Zambroni de Souza. Modeling the integrated natural gas and electricity optimal power flow. 493 In IEEE Power Engineering Society General Meeting, pages 1-7. IEEE, 2007.

494 T. Li, M. Eremia, and Mohammad Shahidehpour. Interdependency of natural gas network and power system security. Power Systems, IEEE 495 Transactions on, 23(4):1817-1824, 2008.

${ }_{496}{ }^{15}$ C. Liu, M. Shahidehpour, Y. Fu, and Z. Li. Security-constrained unit commitment with natural gas transmission constraints. Power Systems, 497 IEEE Transactions on, 24(3):1523-1536, 2009.

${ }_{498}{ }^{16}$ C. M. Correa-Posada and P. Sánchez. Security-constrained optimal power and natural-gas flow. IEEE Transactions on Power Systems, 499 29(4):1780-1787, 2014. 
${ }_{500}{ }^{17} \mathrm{~L}$. Wu and M. Shahidehpour. Optimal coordination of stochastic hydro and natural gas supplies in midterm operation of power systems. 501 Generation, Transmission \& Distribution, 5(5):577-587, 2011.

${ }_{502}{ }^{18}$ C. Sahin, Z. Li, M. Shahidehpour, and I. Erkmen. Impact of natural gas system on risk-constrained midterm hydrothermal scheduling. IEEE 503 Transactions on Power Systems, 26(2):520-531, 2011.

${ }_{504}{ }^{19}$ C. Liu, M. Shahidehpour, and J. Wang. Application of augmented lagrangian relaxation to coordinated scheduling of interdependent 505 hydrothermal power and natural gas systems. Generation, Transmission \& Distribution, 4(12):1314-1325, 2010.

${ }_{506}{ }^{20}$ C. Sahin, M. Shahidehpour, and I. Erkmen. Generation risk assessment in volatile conditions with wind, hydro, and natural gas units. 507 Applied Energy, 96:4-11, 2012.

${ }_{508} 21$ C. Unsihuay, J. W. Marangon-Lima, and A. C. Zambroni de Souza. Short-term operation planning of integrated hydrothermal and natural 509 gas systems. In IEEE Power Engineering Society General Meeting, pages 1410-1416. IEEE, 2007.

${ }_{510} 22$ C. A. Saldarriaga, R. A. Hincapie, and H. Salazar. A holistic approach for planning natural gas and electricity distribution networks. IEEE 511 Transactions on Power Systems, 28(4):4052-4063, 2013.

${ }_{512}{ }^{23}$ Martin Geidl and Göran Andersson. Optimal power flow of multiple energy carriers. Power Systems, IEEE Transactions on, 22(1):145-155, 5132007.

$514{ }^{24}$ R. Z. Ríos-Mercado and C. Borraz-Sánchez. Optimization problems in natural gas transportation systems: A state-of-the-art review. Applied 515 Energy, 147:536-555, 2015.

${ }_{516}{ }^{25}$ C. Borraz-Sanchez. Optimization methods for pipeline transportation of natural gas. PhD thesis, Bergen Univ.(Norway), 2010.

$517{ }^{26}$ B. C. Erdener, K. A. Pambour, R. B. Lavin, and B. Dengiz. An integrated simulation model for analysing electricity and gas systems. 518 International Journal of Electrical Power \& Energy Systems, 61:410-420, 2014.

${ }_{519}{ }^{27}$ C. Liu, M. Shahidehpour, and J. Wang. Coordinated scheduling of electricity and natural gas infrastructures with a transient model for 520 natural gas flow. Chaos: An Interdisciplinary Journal of Nonlinear Science, 21(2):025102, 2011.

${ }_{521}{ }^{28}$ M. Chaudry, N. Jenkins, and G. Strbac. Multi-time period combined gas and electricity network optimisation. Electric power systems $522 \quad$ Research, 78(7):1265-1279, 2008.

${ }_{523}{ }^{29}$ P. Marrin. ISO-like natural gas coordinator among ideas to harmonize gas, power industries. 2015.

${ }_{524}{ }^{30}$ M. Qadrdan, M. Chaudry, J. Wu, N. Jenkins, and J. Ekanayake. Impact of a large penetration of wind generation on the gb gas network. 525 Energy Policy, 38(10):5684-5695, 2010.

${ }_{526}{ }^{31}$ K. Turitsyn, A. Zlotnik, and M. Chertkov. Assessing risk of gas shortage in coupled gas-electricity infrastructures. Submitted to 49th Hawaii 527 International Conference on System Sciences (HICSS), 2016.

${ }_{528} 32$ J. Brouwer, I. Gasser, and M. Herty. Gas pipeline models revisited: Model hierarchies, nonisothermal models, and simulations of networks. 529 Multiscale Modeling \& Simulation, 9:601-623, 2011.

${ }_{530}^{33} \mathrm{P}$. Wong and R. Larson. Optimization of natural-gas pipeline systems via dynamic programming. Automatic Control, IEEE Transactions 531 on, 13(5):475-481, 1968.

${ }_{532}{ }^{34}$ Suming Wu, R.Z. Ros-Mercado, E.A. Boyd, and L.R. Scott. Model relaxations for the fuel cost minimization of steady-state gas pipeline 533 networks. Mathematical and Computer Modelling, 31(23):197 - 220, 2000.

${ }_{534} 35$ Frédéric Babonneau, Yurii Nesterov, and Jean-Philippe Vial. Design and operations of gas transmission networks. Operations Research, $535 \quad 60(1): 34-47,2012$.

${ }_{536}^{36}$ S. Misra, M. W. Fisher, S. Backhaus, R. Bent, M. Chertkov, and F. Pan. Optimal compression in natural gas networks: a geometric 537 programming approach. IEEE Transactions on Control of Network Systems (CONES), 2015.

${ }_{538} 37$ The Williams Transco Pipe Line, http://www.1line.williams.com/Transco/index.html.

${ }_{539}{ }^{38}$ M. Behbahani-Nejad and Y. Shekari. The accuracy and efficiency of a reduced-order model for transient flow analysis in gas pipelines. $540 \quad$ Journal of Petrolium Science and Engineering, 73(13), 2010.

${ }_{541}{ }^{39}$ M. Herty, J. Mohringb, and V. Sachersa. A new model for gas flow in pipe networks. Math. Meth. Appl. Sci., 33:845-855, 2010.

${ }_{542}^{40}$ CRANE. Flow of fluids: Through valves, fittings and pipe. Technical paper 410M, Crane Company, New York, 1982.

${ }_{543} 41$ S. Mokhatab, W. A. Poe, and J. G. Speight. Handbook of Natural Gas Transmission and Processing. Gulf Professional Publishing, Houston, 5442006.

$545{ }^{42}$ Conrado Borraz-Sánchez. Optimization Methods for Pipeline Transportation of Natural Gas. PhD thesis, Department of Informatics, $546 \quad$ University of Bergen, Norway, October 2010. 